\title{
1.38 MW peak power dual-loss modulated sub-nanosecond green laser with EO and Graphene
}

\author{
Wenjing Tang, Jia Zhao, Kejian Yang, Shengzhi Zhao ${ }^{*}$, Guiqiu Li, Dechun Li, Tao Li, Wenchao \\ Qiao \\ School of information science and engineering, Shandong University, 27 Shanda South Road,
}

Jinan 250100, China

*Corresponding author: shengzhi_zhao@sdu.edu.cn

\begin{abstract}
By simultaneously employing electro-optic (EO) modulator and Graphene saturable absorber (SA) in a dual-loss-modulated Q-switched and mode-locking (QML) Nd:Lu $\mathrm{Lu}_{0.15} \mathrm{Y}_{0.85} \mathrm{VO}_{4} /$ KTP green laser, the sub-nanosecond single mode-locking green laser is demonstrated with high peak power, low repetition rate and high stability. The monolayer and 3-layer graphene sheets grown by chemical vapor deposition (CVD) method were used as SAs in the experiment. When the pump power reached $10.72 \mathrm{~W}$, the maximum peak power obtained from the doubly QML laser with EO and monolayer graphene-SA was $1.38 \mathrm{MW}$, corresponding to a pulse duration of $480 \mathrm{ps}$. The shortest pulse width of $340 \mathrm{ps}$ was obtained with a 3-layer graphene-SA.
\end{abstract}

Keywords: solid-state; dual-loss-modulated; graphene; sub-nanosecond.

\section{Introduction}

With the extremely short pulse duration and high peak power, diode-pumped solid-state ultrafast laser sources have become one of the most active fields in the laser research. Especially in the fields of underwater optical communication, ocean exploration, laser show and industrial materials processing, the ultrafast green lasers are always at the center of an increasing research effort [1-4]. As we know, ultrafast green pulses are mostly obtained from the $1064 \mathrm{~nm}$ pulsed lasers by inserting a double frequency crystal in the cavity [5,6]. For this type of green lasers, a high pulse peak power at the fundamental wavelength can apparently improve the intracavity second harmonic (SH) conversion efficiency. Nevertheless, current continuous wave mode-locking technology still suffer from drawbacks in terms of obtaining pulses with ultrashort duration and high pulse energy simultaneously, e.g., the high pulse repetition rates can reduce the energy per pulse, resulting in small SH conversion efficiency. Thus, ever growing research on novel materials, new technologies are motivated by the limitation of CW mode-locking.

To generate ultrashort pulses with high pulse energy, the dual-loss-modulation Q-switched mode-locking (QML) technology is really a better choice [7, 8]. By simultaneously employing the active modulator and the saturable absorber (SA), the dual-loss-modulated QML regime can apparently compress the pulse duration of the Q-switched envelope and enhance the pulse stability. If the pulse width of the Q-switched envelope can be compressed shorter than the cavity roundtrip transmit time, there will be only one mode-locking pulse inside. Then, the single mode-locking operation of a dual-loss-modulated QML laser can be realized. In this case, the entire energy of Q-switched envelope will gather on this single mode-locked pulse, resulting in a large energy per pulse. In 2012, a dual-loss-modulation $\mathrm{QML} \mathrm{YVO}_{4} / \mathrm{Nd}: \mathrm{YVO}_{4}$ laser with electro-optic (EO) and 
SESAM to generate stable sub-nanosecond single mode-locking pulses has been demonstrated for the first time [9].

Furthermore, the saturable absorber (SA) has very important influence on the mode-locking operation. Besides technical challenges, the development of ultrafast lasers is also largely dependent on the development of SA. Over the past two decades, SESAMs have been widely used in passive mode-locked green lasers $[5,6,10]$. However, these SA mirrors require very complex and costly fabrication processes. The saturable absorption bandwidth is also limited by the Bragg mirrors. Alternative materials with low-cost and simple fabrication methods are therefore in great request. The experimental realization of graphene has led to an intense investigation of the fundamental properties and potential applications of 2D nanomaterials. In recent years, several novel SAs have been developed for ultrafast laser operation, such as graphene [11, 12], carbon nanostructures [13], topological insulators[14], metal dichalcogenides (s-TMDs) [15]. Among these SA materials, graphene has been attracting much attention owing to its fascinating physical properties, ideal optical characteristics, mechanical and environmental robustness [16-18]. Most importantly, due to the ultrafast carrier dynamics and large absorption of incident light per layer $(\alpha=2.3 \%)$, graphene as a fast saturable absorber owns a wide-band operation potential which successfully solves the problem of bandwidth limitation [19]. To date, various laser applications have been realized by using graphene or graphene based novel devices [20-22]. However, the generation of single mode-locked pulses from the graphene-based dual-loss-modulation QML green laser has not been reported experimentally until now.

In this paper, a dual-loss-modulation QML green laser with an EO modulator and graphene-SA is presented. Mixed crystal $\mathrm{Nd}: \mathrm{Lu}_{0.15} \mathrm{Y}_{0.85} \mathrm{VO}_{4}$ is used as the gain medium for its better optical properties [23, 24]. Compared with the single vanadate crystal, mixed vanadate crystal is much easier to get larger pulse energy and shorter pulse duration [25]. Two kinds of graphene SAs (monolayer and 3-layer graphene) are used in the experiment. Stable single mode-locked pulses with high peak power can be obtained. Under the repetition rate of $1 \mathrm{kHz}$ and the pump power of $10.72 \mathrm{~W}$, the shortest pulse duration obtained with 3-layer graphene-SA is about $340 \mathrm{ps,}$ corresponding to a peak power of $1.2 \mathrm{MW}$. The maximum peak power of $1.38 \mathrm{MW}$ is obtained with monolayer graphene-SA.

\section{Experiment setup}

The schematic configuration of the dual-loss-modulated QML Nd:Lu ${ }_{0.15} \mathrm{Y}_{0.85} \mathrm{VO}_{4}$ green laser with EO and graphene-SA is shown in Fig. 1. To obtain stable and efficient Q-switching and mode locking simultaneously, a Z-type cavity which consists of four mirrors is well designed. The total physical cavity length is $142.5 \mathrm{~cm}$ while the lengths of three cavity arms are 55.5, 78, and $9 \mathrm{~cm}$, respectively. The flat mirror M1, anti-reflection (AR) coated at $808 \mathrm{~nm}$ on both surface and high-reflection (HR) coated at 1064 and $532 \mathrm{~nm}$ on the inside surface, is adopted as the input mirror. The concave mirror M2 with a radius of curvature (ROC) of $500 \mathrm{~mm}$ and the flat mirror M4, acting as the resonator mirrors, are both HR coated at $1064 \mathrm{~nm}$ and $532 \mathrm{~nm}$. The output mirror M3 with a ROC of $150 \mathrm{~mm}$ is HR coated at $1064 \mathrm{~nm}$ and AR coated at $532 \mathrm{~nm}$. The pump source is a commercial fiber coupled Laser diode (FAP-I system, Coherent Inc., USA) operating at $808 \mathrm{~nm}$ with a fiber core diameter of $400 \mu \mathrm{m}$. By an optical coupled system, the pump beam with a spot radius of $200 \mu \mathrm{m}$ is collimated and focused into the laser gain medium. The laser gain 
medium is an a-cut mixed laser crystal $\mathrm{Nd}: \mathrm{Lu}_{0.15} \mathrm{Y}_{0.85} \mathrm{VO}_{4}$ with the dimension of $3 \times 3 \times 10 \mathrm{~mm} 3$ and Nd-doping concentration of 0.38 at.\%. Both surfaces of the laser crystal are AR coated at 808 and $1064 \mathrm{~nm}$. As the frequency-doubling crystal, KTP with the dimension of $3 \times 3 \times 8 \mathrm{~mm} 3$ is cut for type-II phase matching at $1064 \mathrm{~nm}\left(\theta=90^{\circ}, \varphi=23.2^{\circ}\right)$ and AR coated at 1064 and $532 \mathrm{~nm}$ on both facets. A thermo-electric cooler is used to efficiently dissipate the heat deposition in the laser crystal and KTP, maintaining at a low temperature of $12{ }^{\circ} \mathrm{C}$ during the experiment. With a polarizer and a $\lambda / 4$ plate, an EO modulator (BBO crystal, the repetition rate can be modulated from 1 to $5 \mathrm{kHz}$ ) is employed as the active modulator while two kinds of graphene sheets (monolayer and 3-layer graphene) are used as SAs. The output powers were measured by a PM100D Energy/Power Meter (Thorlabs Inc., USA). The pulse characteristics were recorded by a 16G digital oscilloscope (Agilent DSO-X91604A, $80 \mathrm{G}$ samples/s sampling rate, Tektronix Inc., USA) and a fast pin photodiode detector (New Focus 1414) with a rise time of 14 ps.

Fig. 1 Schematic configuration of the dual-loss-modulated QML Nd: $\operatorname{Lu}_{0.15} \mathrm{Y}_{0.85} \mathrm{VO}_{4}$ laser.

\section{Results and Discussion}

\subsection{Characterization of Graphene-SA}

The graphene employed in our experiment was purchased from JCNano Co. Ltd., China. The monolayer graphene was grown by chemical vapor deposition (CVD) technology. The 3-layers graphene film was obtained by stacking the monolayer graphene layer-by-layer. The size of the graphene film transferred onto the quartz substrate was approximately $1.5 \times 1.5 \mathrm{~cm}^{2}$. The Raman spectra of the two graphene films were measured using a Horiba Jobin Yvon Raman system with a pump laser at $514 \mathrm{~nm}$ and shown in Fig. 2(a). For the monolayer graphene, the Raman spectrum shows two typical peaks: $G$ and $2 D$ at $1596.5 \mathrm{~cm}^{-1}$ and $2652.7 \mathrm{~cm}^{-1}$ with line width of $17.5 \mathrm{~cm}^{-1}$ and $38 \mathrm{~cm}^{-1}$, respectively. And the $\mathrm{G}$ and 2D peaks for 3-layer graphene are at $1599 \mathrm{~cm}^{-1}$ and 2673 $\mathrm{cm}^{-1}$ with line width of $32 \mathrm{~cm}^{-1}$ and $74 \mathrm{~cm}^{-1}$, respectively. The broaden line width of the 2D-band was mainly due to the multi-layer stacks [26]. The D-band is hardly observed in the Raman spectrum of the monolayer graphene, suggesting a very high crystalline quality of graphene SA is prepared. For the 3-layer graphene, an increase of defects results in an increase of the D-band $\left(\sim 1345 \mathrm{~cm}^{-1}\right)$ intensity.

To estimate parameters important for Q-switching and mode-locking, such as saturation intensities, modulation depths and nonsaturable losses, the nonlinear transmission characteristics of the monolayer and 3-layer graphene SAs were measured at $1.06 \mu \mathrm{m}$ using the balanced twin-detector measurement technique and shown in the Fig. 2(b). Fig. 2(c) shows the experimental setup for saturable absorption measurement, which was the same with the one described in ref [27].The laser source is a homemade mode-locking laser which can generate picosecond laser pulses with a repetition rate of $40 \mathrm{MHz}$. M1, M2 and M3 are the adjustable attenuator, beam splitter and focal lens, respectively. By fitting the data, the modulation depths of $1.6 \%$ and $3.4 \%$, saturation intensities of $0.56 \mathrm{MW} / \mathrm{cm}^{2}$ and $0.73 \mathrm{MW} / \mathrm{cm}^{2}$, and the nonsaturable losses of $1.7 \%$ and $8.1 \%$ for the monolayer and 3-layer graphene SAs, respectively. The results show that the longer optical absorption path, nonuniformity and the scattering of the 3-layer graphene contribute to a larger optical loss in both linear and nonlinear regimes. 
Fig. 2 (a) Raman spectra; (b) Nonlinear transmission of graphene-SAs; (c) Experimental setup for saturable absorption measurement.

\subsection{Experimental results and discussion}

By a careful adjustment to the dual-loss-modulated QML Nd:Lu ${ }_{0.15} \mathrm{Y}_{0.85} \mathrm{VO}_{4}$ green laser with graphene SAs, stable QML regimes can be observed. Fixing the active modulation frequency of $\mathrm{EO}$ at $1 \mathrm{kHz}$, the performance of the dual-loss-modulated QML green laser with the monolayer and 3-layer graphene based SA were investigated and compared.

The average output powers versus the incident pump power are displayed in Fig. 3. The average powers output by laser with monolayer graphene-SA are much higher than that generated with 3-layer graphene-SA. At the pump power of $10.72 \mathrm{~W}$, the maximum output powers of the dual-loss-modulated green laser with monolayer and 3-layer graphene SA are $661 \mathrm{~mW}$ and 407 $\mathrm{mW}$, respectively. In order to verify the robustness of this green laser with the graphene-SA, the fluctuation of the maximum output power is measured. At the pump power of $10.72 \mathrm{~W}$, the fluctuation of the maximum average output power $\Delta \mathrm{P}$ are less than $2.9 \%$ and $3.1 \%$ for the lasers with the monolayer and 3-layer graphene-SAs, respectively, showing the high stability of this single mode-locked laser. The corresponding spectrum of the pulsed laser at $532.3 \mathrm{~nm}$ is measured and shown in Fig.3 (insert).

Fig. 3 Average output power versus the pump power for different SAs. Insert: Output laser spectrum.

Using a digital oscilloscope and a fast pin photodiode detector, the pulse characteristics can be observed intuitively. The pulse widths versus the pump power for different graphene-SAs are shown in Fig. 4. From the figure we can see that the green laser operates at the QML stage (solid symbols in Fig. 4) with low pump power. When the pump power reaches the threshold power of getting the stable sub-nanosecond single mode-locking pulses, the laser operates at the single mode-locking stage (open symbols in Fig. 4). The threshold pump powers for the dual-loss-modulated laser with monolayer and 3-layer graphene-SAs are $9.84 \mathrm{~W}$ and $8.08 \mathrm{~W}$, respectively. The pulse widths of both the two stages decrease rapidly with the increase of the pump power, leading to a reduction in the number of mode-locking pulses underneath a Q-switched envelope at the QML stage [shown in Fig. 4 (insert)]. From Fig. 4 we also can find that the pulse duration generated by the 3-layer graphene-SA is shorter than that obtained by the monolayer graphene-SA. This is mainly caused by the larger nonsaturable loss and modulation depth of 3-layer graphene-SA which can help to compress the pulse width of the Q-switched envelope. The minimum pulse durations of $480 \mathrm{ps}$ and $340 \mathrm{ps}$ are generated by the monolayer and 3-layer graphene-SAs at the pump power of $10.72 \mathrm{~W}$, respectively.

Fig. 4. Dependence of the pulse widths versus the pump power for different SAs. Insert: Number of mode-locked pulses underneath a Q-switched envelop versus the pump power. Solid symbols: the QML stage; Open symbols: the single mode-locking stage.

In order to observe the variation of mode-locking pulses underneath the Q-switched envelope and the stability of the sub-nanosecond pulses more directly and conveniently, the oscilloscope trace of the dual-loss-modulated QML pulses for two different SAs is shown in the Fig. 5. When 
the pump power is $6.28 \mathrm{~W}$, there are four and three mode-locking pulses underneath the Q-switched envelope for monolayer and 3-layer graphene-SAs, respectively. When the pump power is raised to $9.84 \mathrm{~W}$, we can see that both the QML green laser systems with monolayer and 3-layer graphene-SAs operate in the single mode-locking state. The extended pulse temporal behaviors of two shortest single mode-locking pulses with pulse widths of $480 \mathrm{ps}$ and $340 \mathrm{ps}$ are also illustrated in the figure at the pump power of $10.72 \mathrm{~W}$. From the figure it can be seen that the single mode-locked pulses have asymmetry structures. A very small pulse appears after the large single mode-locked pulse. This may be because that the open time of EO switch is longer than the pulse duration of the large single mode-locking pulse, and there is still gain enough in the laser crystal to support the small pulses after the first large pulse generated in the cavity. Since almost 98\% energy of the Q-switched envelope was concentrated into the main large mode-locking pulse, the condition of a single mode-locking pulse operation was considered to have been achieved. The temporal single mode-locked pulse trains generated with different SAs are shown on the far right of Fig. 5. At the pump power of $10.72 \mathrm{~W}$, the pulse to pulse amplitude fluctuation was estimated about $3 \% \mathrm{rms}$, which demonstrates the high stability of the single mode-locking pulses. To check the thermal stability and mechanical strength of the dual-loss-modulated QML Nd:Lu $u_{0.15} \mathrm{Y}_{0.85} \mathrm{VO}_{4}$ green laser, the experiment was kept operating for about 6 hours every day during five days. The laser system ran at a stable sub-nanosecond single mode-locking status during the whole procedure and no damage of the graphene sheets was observed. Compared to most of the saturable absorber materials, the super thermal conduction property of graphene is beneficial for the high peak power and long-playing single mode-locking operation with high stability [16, 28].

Fig. 5. Pulse shapes of the dual-loss-modulated green laser at different pump powers. (a): monolayer graphene; (b): 3-layer graphene.

According to the average output power, the pulse repetition rate and the number of the mode-locking pulses underneath a Q-switched envelope, the average mode-locking pulse energy can be obtained, which is shown in Fig. 6. At the QML stage, one can see that the pulse energies generated by the monolayer graphene-SA are almost nearly the same with that obtained with the 3-layer graphene-SA. When two lasers all operate at single mode-locking status, the larger envelope energy of laser with the monolayer graphene-SA yields much higher mode-locking pulse energy. The highest single pulse energies are 661 and $407 \mu \mathrm{J}$ with monolayer and 3-layer graphene-SAs at the pump power of $10.72 \mathrm{~W}$, respectively.

Fig. 6. Dependence of the mode-locking pulse energy on the pump power. Solid symbols: the QML stage; Open symbols: the single mode-locking stage.

By dividing the per-pulse energy with the pulse duration of the single mode-locking pulse, the peak powers of the dual-loss-modulated QML laser can be obtained, as depicted in Fig. 7. We can see that the peak powers grow almost exponentially as the rise of the pump power. At the single mode-locking stage, although the laser with monolayer graphene-SA yields a larger pulse width, it also has advantages in generating high peak power for its large pulse energy. The highest peak powers are up to $1.38 \mathrm{MW}$ and 1.2 MW for the monolayer and 3-layer graphene-SAs at the pump power of $10.72 \mathrm{~W}$. 
Fig. 7. Dependence of the mode-locking pulse peak power on the pump power. Solid symbols: the QML stage;

Open symbols: the single mode-locking stage.

By employing the 90.0/10.0 scanning-knife-edge method, the beam quality for the high peak power sub-nanosecond lasers was measured. The beam quality factors $\left(\mathrm{M}^{2}\right)$ in the horizontal and longitudinal planes are 1.87/1.56 for the laser with monolayer graphene-SA, and 1.84/1.55 for the laser with 3-layer graphene-SA.

\section{Conclusions}

In conclusion, high-quality graphene-SAs (monolayer and 3-layer graphene) were successfully used for the generation of the sub-nanosecond single mode-locking green pulses. At the pump power of $10.72 \mathrm{~W}$, the maximal peak power of sub-nanosecond green pulses are $1.38 \mathrm{MW}$ and 1.2 MW, which correspond to the shortest pulse duration of 480 and $340 \mathrm{ps}$ for monolayer and 3-layer graphene-SAs, respectively. The experimental results indicate that the graphene-SA can be applied to produce high-peak-power, sub-nanosecond single mode-locking pulses.

\section{Acknowledgments}

This work is partially supported by the National Science Foundation of China (61378022) and the Fundamental Research Funds of Shandong University (2014JC032).

\section{Reference}

[1] T. Wiener and S. Karp, The Role of Blue/Green Laser Systems in Strategic Submarine Communications, IEEE Transactions on Communications 28(9) (1980) 1602-1607.

[2] A. J. Antończak, P. Kozioł, A. T. Wąż, J. Z. Sotor, G. Dudzik, P. R. Kaczmarek, and K. M. Abramski , Underwater green laser vibrometry, AIP Conf. Proc. 1457 (2012) 251-256.

[3] J. Y. Cheng, M. H. Yen, T. H. Young, Crack-free micromachining on glass using an economic Q-switched 532 nm laser, J. Micromech. Microeng. 16(11) (2006) 2420.

[4] J. J. Chang, B. E. Warner, E. P. Dragon, M. W. Martinez, Precision micromachining with pulsed green lasers, J. Laser Appl. 10 (1998) 285-291.

[5] X. Wang, M. Li, Continuous-wave passively mode-locked Nd:YVO $4 / \mathrm{KTP}$ green laser with a semiconductor saturable absorber mirror, Laser Phys. 20 (2010) 733-736.

[6] L. Li, J. Liu, M. Liu, S. Liu, F. Chen, W. Wang and Y. Wang, $532 \mathrm{~nm}$ continuous wave mode-locked Nd:GdVO 4 laser with SESAM, Laser Phys. Lett. 6(2) (2009) 113-116.

[7] T. Li, S. Zhao, et al., Dual-loss-modulated Q-switched and mode-locked $\mathrm{YVO}_{4} / \mathrm{Nd} \mathrm{YVO}_{4} / \mathrm{KTP}$ green laser with EO and $\mathrm{Cr}^{4+}$ :YAG saturable absorber, Opt. Exp. 18 (2010) 10315-10322.

[8] T. Feng, S. Zhao, K. Yang, G. Li, D. Li, J. Zhao, B. Zhao, Y. Wang, Intracavity-frequency-doubled Q-switched and mode-locked $\mathrm{Nd}: \mathrm{Lu}_{0.15} \mathrm{Y}_{0.85} \mathrm{VO}_{4} / \mathrm{KTP}$ green laser with AO modulator and central SESAM, Optik 125 (2014) 2739-2744.

[9] G. Zhang, S. Zhao, G. Li, K. Yang, D. Li, K. Cheng, Y. Wang, Stable sub nanosecond pulse generation from dual-loss-modulated $\mathrm{QML} \mathrm{YVO}_{4} / \mathrm{Nd}: \mathrm{YVO}_{4}$ laser with $\mathrm{EO}$ and middle SESAM, Opt. Commun. 285(24) (2012) 5347-5350.

[10] W. Wang, J. Liu, F. Chen, L. Li, Y. Wang, 532-nm picosecond pulse generated in a passively mode-locked Nd: $\mathrm{YVO}_{4}$ laser, Chinese Opt. Lett. 7(8) (2009) 706-708.

[11] W. Cho, J. Kim, H. Lee, S. Bae, B. Hong, S. Choi, I. Baek, K. Kim, D. Yeom, and F. 
Rotermund, High-quality, large-area monolayer graphene for efficient bulk laser mode-locking near $1.25 \mu \mathrm{m}$, Opt. Lett. 36 (2011) 4089-4091.

[12] Q. Bao, H. Zhang, Y. Wang, Z. Ni, Y. Yan, Z. Shen, K. Loh, and D. Tang, Atomic-layer graphene as a saturable absorber for ultrafast pulsed lasers, Adv. Funct. Mater. 19(19) (2009) 3077-3083.

[13] Z. Sun, T. Hasan, A.C. Ferrari, Ultrafast lasers mode-locked by nanotubes and graphene Physica E 44 (2012) 1082-1091.

[14] C. Zhao, Y. Zou, Y. Chen, Z. Wang, S. Lu, H. Zhang, S. Wen, and D. Tang, Wavelength-tunable picosecond soliton fiber laser with Topological Insulator: $\mathrm{Bi}_{2} \mathrm{Se}_{3}$ as a mode locker, Opt. Exp. 20 (2012) 27888-27895.

[15] H. Zhang, S. B. Lu, J. Zheng, J. Du, S. C. Wen, D. Y. Tang, and K. P. Loh, Molybdenum disulfide $\left(\mathrm{MoS}_{2}\right)$ as a broadband saturable absorber for ultra-fast photonics, Opt. Exp. 22 (2014) 7249-7260.

[16] A. A. Balandin, S. Ghosh, W. Bao, I. Calizo, D. Teweldebrhan, F. Miao, C. N. Lau, Superior Thermal Conductivity of Single-Layer Graphene, Nano Lett. 8 (2008) 902-907.

[17] R. R. Nair, P. Blake, A. N. Grigorenko, K. S. Novoselov, T. J. Booth, T. Stauber, N. M. R. Peres, and A. K. Geim, Fine Structure Constant Defines Visual Transparency of Graphene, Science 320 (2008) 1308.

[18] F. Bonaccorso, Z. Sun, T. Hasan, and A.C. Ferrari, Graphene photonics and optoelectronics, Nature Phot. 4 (2011), 611-622.

[19] Z. Sun, T. Hasan, F. Torrisi, D. Popa, G. Privitera, F. Wang, F. Bonaccorso, D. Basko, and A. Ferrari, Graphene mode-locked ultrafast laser, ACS Nano. 4 (2010) 803-810.

[20] M. Lorenzoni , F. Brandi , S. Dante , A. Giugni , B. Torre, Simple and effective graphene laser processing for neuron patterning application, Scientific Reports 3 (2013) 1954.

[21] D. G. Purdie, D. Popa, V. J. Wittwer, Z. Jiang, G. Bonacchini, F. Torrisi, S. Milana, E. Lidorikis, and A. C. Ferrari, Few-cycle pulses from a graphene mode-locked all-fiber laser, Appl. Phys. Lett. 106 (2015) 253101.

[22]S. Chakraborty, O. P. Marshall, T. G. Folland, Y.J. Kim, A. N. Grigorenko, K. S. Novoselov, Gain modulation by graphene plasmons in aperiodic lattice lasers, Science 351 (2016) 246-248.

[23] J. Liu, Z. Wang, X. Meng, Z. Shao, B. Ozygus, A. Ding, and H. Weber, Improvement of passive Q-switching performance reached with a new Nd-doped mixed vanadate crystal Nd: $\mathrm{Gd}_{0.64} \mathrm{Y}_{0.36} \mathrm{VO}_{4}$, Opt. Lett. 28(23) (2003) 2330-2332.

[24] H. Yu, H. Zhang, Z. Wang, J. Wang, Y. Yu, D. Tang, G. Xie, H. Luo, and M. Jiang, Passive mode-locking performance with a mixed $\mathrm{Nd}: \mathrm{Lu}_{0.5} \mathrm{Gd}_{0.5} \mathrm{VO}_{4}$ crystal, Opt. Exp. 17(5) (2009) 3264-3269.

[25] L. Wu, S. Zhao, D. Li, G. Li, K. Yang, J. Zhao, B. Zhao, Performance of doubly Q-switched and mode-locked $\mathrm{Nd}: \mathrm{Lu}_{0.33} \mathrm{Y}_{0.37} \mathrm{Gd}_{0.3} \mathrm{VO}_{4}$ and $\mathrm{Nd}: \mathrm{Lu}_{0.5} \mathrm{Y}_{0.5} \mathrm{VO}_{4}$ lasers with acousto-optic modulator and GaAs saturable absorber, Opt. Commun. 304(1) (2013) 1-6.

[26] A. C. Ferrari, J. C. Meyer, V. Scardaci, C. Casiraghi, M. Lazzeri, F. Mauri, S. Piscanec, D. Jiang, K. S. Novoselov, S. Roth, A. K. Geim, Raman Spectrum of Graphene and Graphene Layers, PRL 97 (2006) 187401.

[27] R. Zhou, P. H. Tang, Y. Chen, S. Q. Chen, C. J. Zhao, H. Zhang, and S. C. Wen, Large-energy, narrow-bandwidth laser pulse at $1645 \mathrm{~nm}$ in a diode-pumped Er:YAG solid-state laser passively Q-switched by a monolayer graphene saturable absorber, Appl. Opt. 53 (2014) 254-258 
[28] J. H. Seol, I. Jo, A. L. Moore, L. Lindsay, Z. H. Aitken, M. T. Pettes, X. Li, Z. Yao, R. Huang, D. Broido, N. Mingo, R. S. Ruoff, L. Shi, Two-Dimensional Phonon Transport in Supported Graphene, Science 328 (2010) 213-216. 


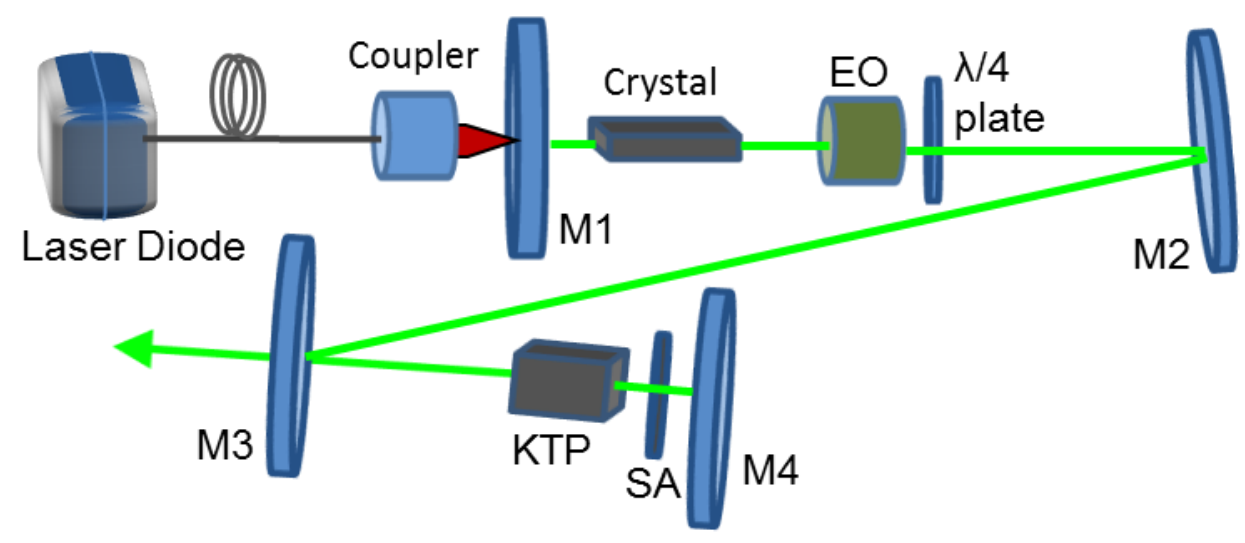






Wavenumber $\left(\mathrm{cm}^{-1}\right)$
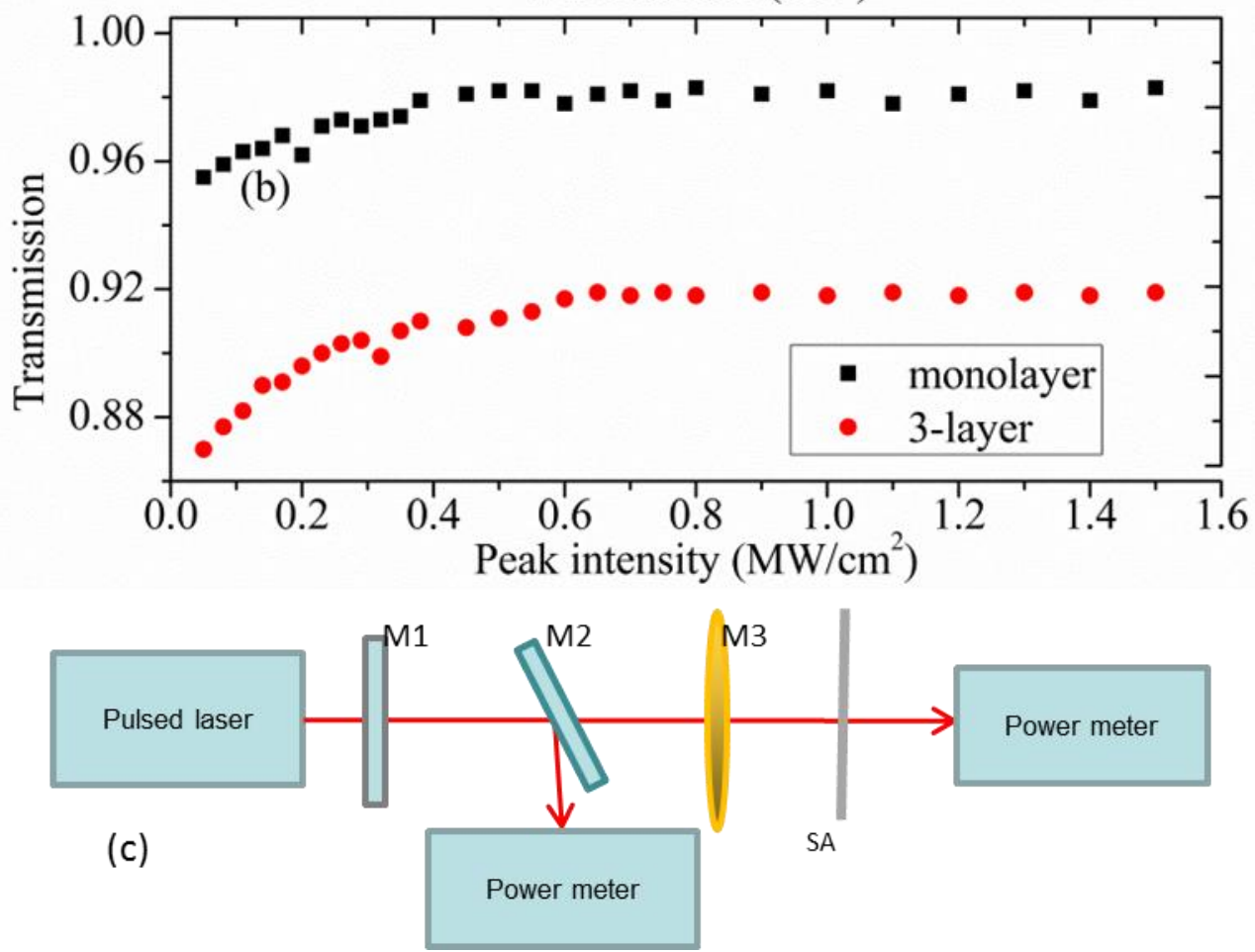


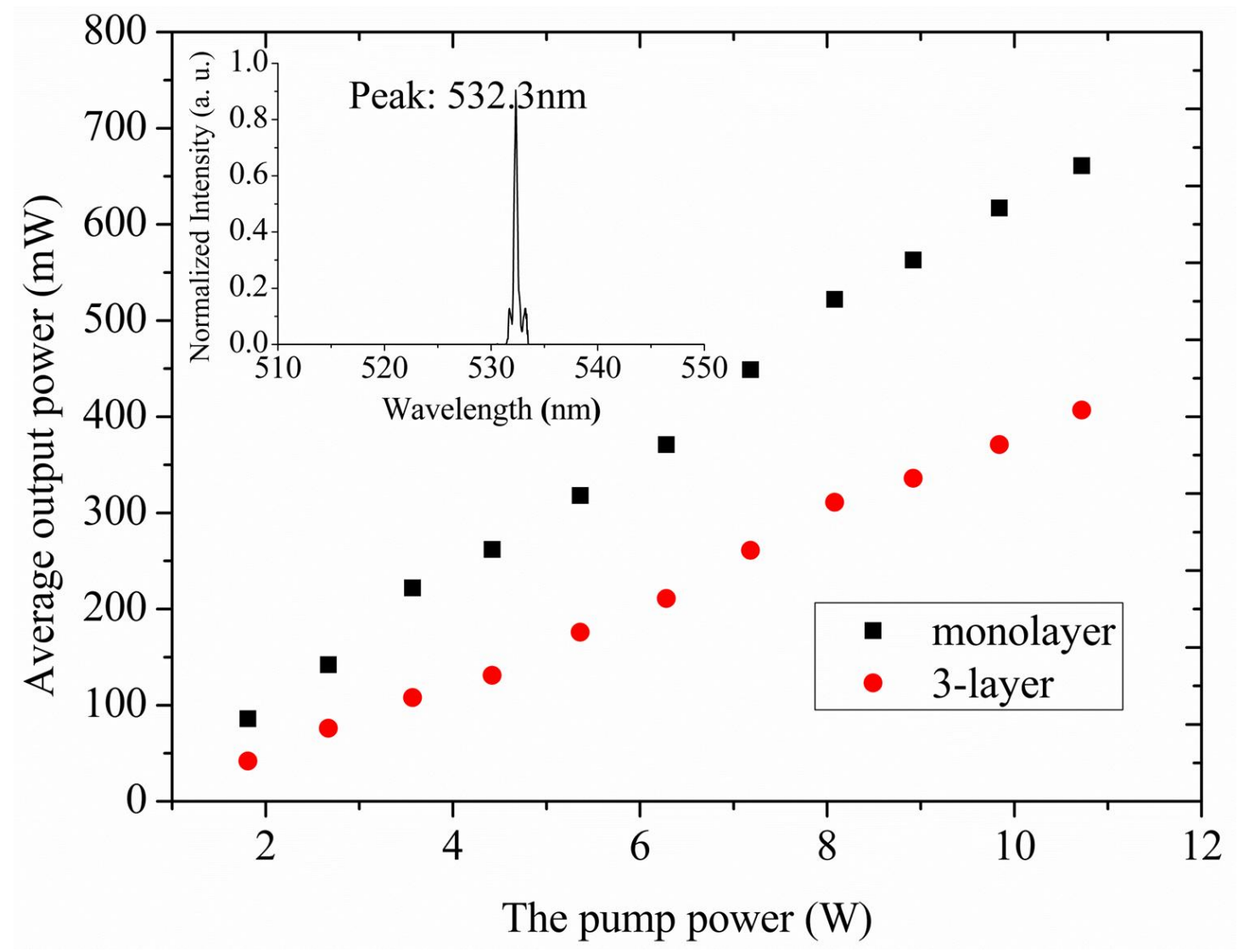




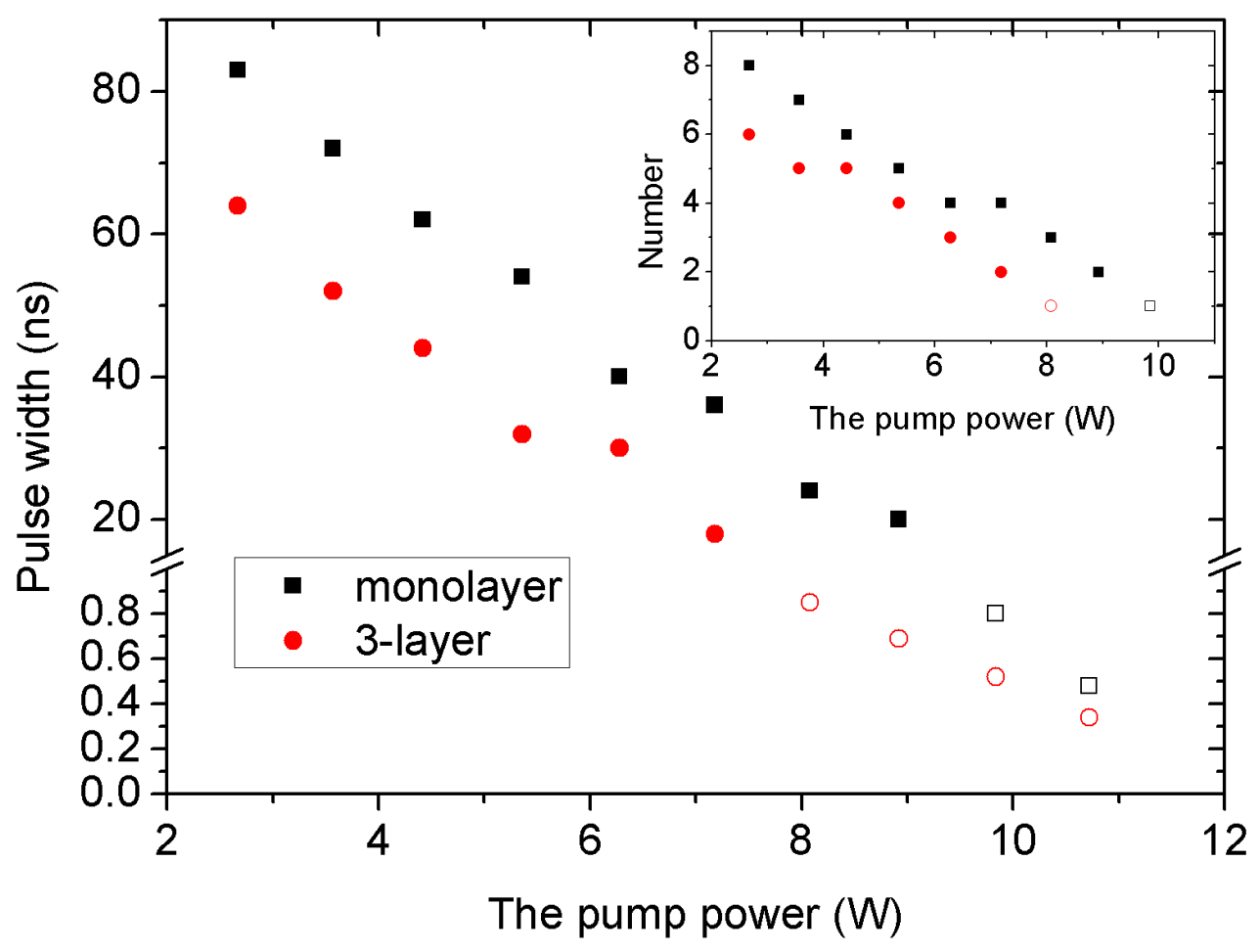


(a)

\begin{tabular}{|c|c|c|c|c|c|c|c|}
\hline $20 \mathrm{~ns} / \mathrm{div}$ & $6.28 \mathrm{~W}$ & $20 \mathrm{~ns} / \mathrm{div}$ & $9.84 \mathrm{~W}$ & 500ps/div & $10.72 W$ & $1 \mathrm{~ms} / \mathrm{div}$ & $10.72 \mathrm{~W}$ \\
\hline & & & & & & & \\
\hline $20 \mathrm{~ns} / \mathrm{div}$ & $6.28 \mathrm{~W}$ & 20ns/div & $9.84 \mathrm{~W}$ & $500 \mathrm{ps} / \mathrm{div}$ & $10.72 W$ & $1 \mathrm{~ms} / \mathrm{div}$ & $10.72 \mathrm{~W}$ \\
\hline & & & & 3 & & & \\
\hline & & & & & & & \\
\hline & & & & & & & \\
\hline
\end{tabular}




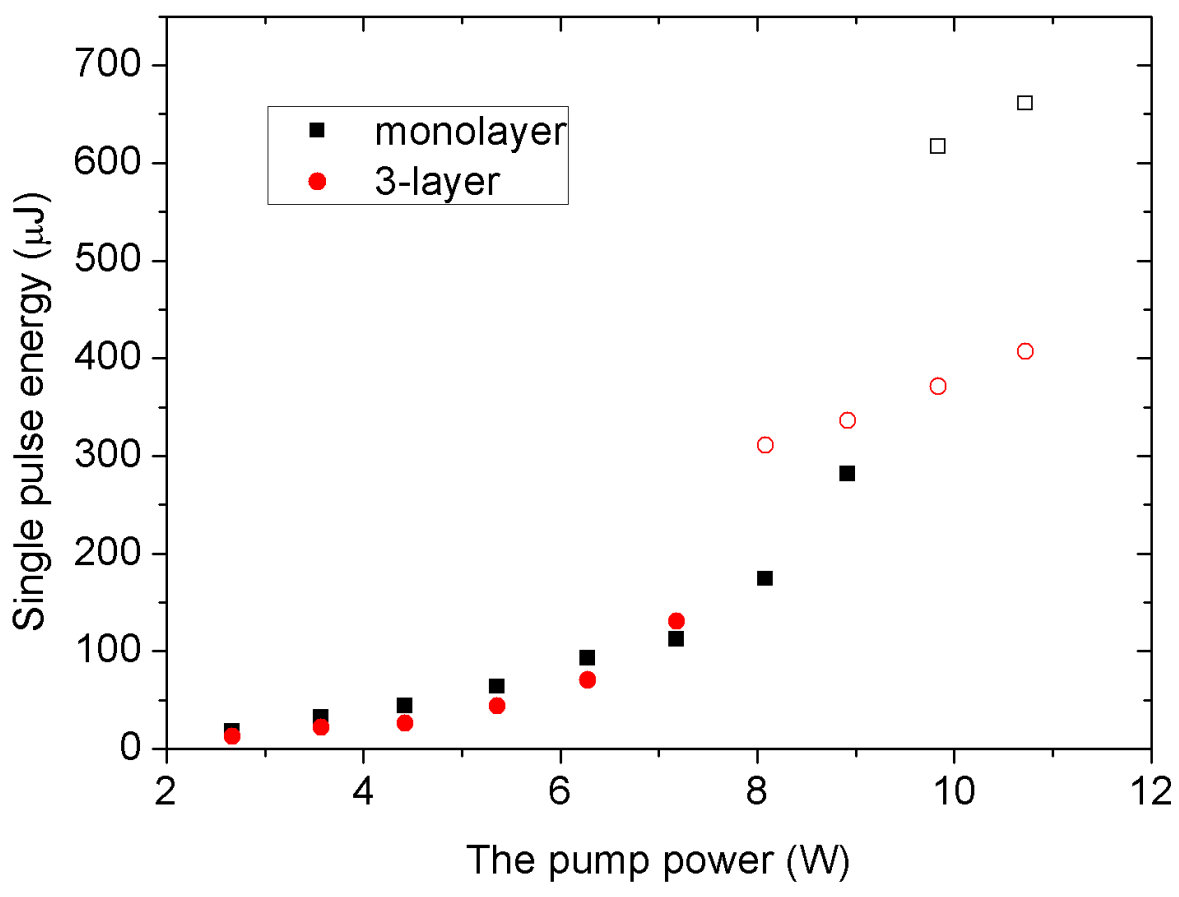




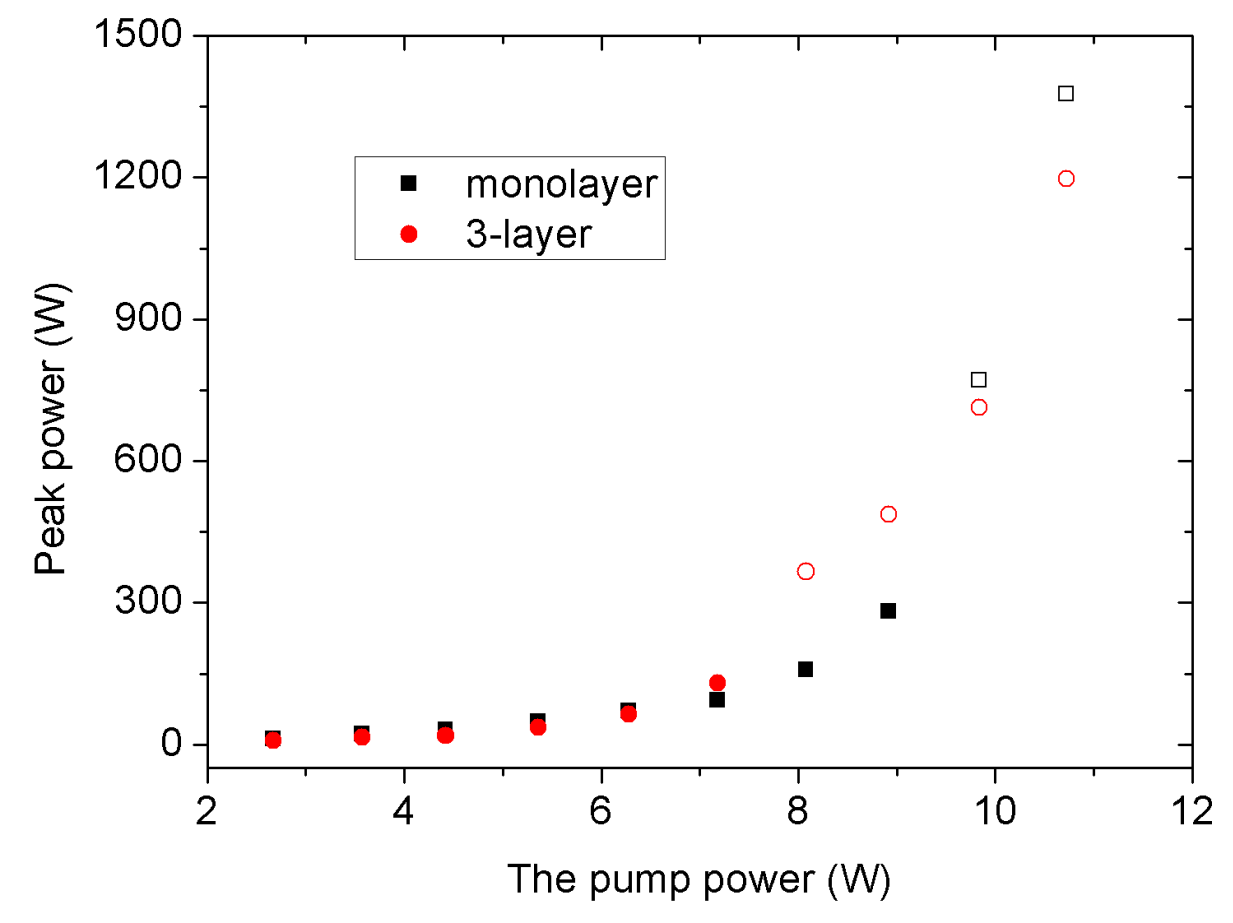

